Check for updates

Cite this: New J. Chem., 2019, 43,3146

Received 9th October 2018,

Accepted 16th January 2019

DOI: $10.1039 / c 8 n j 05133 j$

rsc.li/njc

\section{Facile and green synthesis of palladium nanoparticles loaded into cyclodextrin nanofibers and their catalytic application in nitroarene hydrogenation $\dagger$}

\begin{abstract}
Asli Celebioglu, ${ }^{a}$ Fuat Topuz (D) ${ }^{a}$ and Tamer Uyar (D)*ab
Herein, catalytically active cyclodextrin (CD) nanofibers loaded with in situ formed Pd nanoparticles (Pd-NPs) were prepared by solution electrospinning. Cyclodextrin (CD) acted as a reducing agent and catalyzed the formation of noble metal nanoparticles, e.g., palladium ( $\mathrm{Pd}$ ) over the reduction from $\mathrm{Pd}^{2+}$ to metallic $\mathrm{Pd}^{0}$, without requiring any other reducing agent. Nanofibers were produced by the electrospinning of CD molecules from aqueous and DMF solutions containing two different Pd loadings ( 1 and 2 wt\% with respect to $C D$ ). The electrospinning of these solutions could give rise to bead-free CD nanofibers whose diameters showed variations depending on the solvent-type and the Pd content used: the nanofibers electrospun from DMF solutions were smaller in diameter than those produced from aqueous solutions. Furthermore, increasing Pd loading decreased the nanofiber diameter. TEM, HRTEM, STEM and SAED analyses confirmed the presence of homogeneously distributed polycrystalline Pd-NPs in the size range of 3-5 $\mathrm{nm}$ throughout the nanofiber matrix. XPS experiments demonstrated the presence of a higher proportion of metallic $\mathrm{Pd}^{0}$ atoms owing to the efficient reduction of $\mathrm{Pd}^{2+}$ by $\mathrm{CD}$ molecules. Lastly, the catalytic activity of the nanocomposite nanofibers was explored by the reduction of a nitroarene compound, $p$-nitrophenol (PNP), to $p$-aminophenol (PAP), and high catalytic activity of the nanofibers was observed.
\end{abstract}

\section{Introduction}

There is growing interest in engineering functional electrospun nanofibers with tunable structural properties due to their use in a wide spectrum of applications. ${ }^{1-3}$ Particularly, catalytically active nanofibers have gained considerable interest owing to their handful and reusable structure. ${ }^{4-6}$ In this regard, various strategies have been developed for the engineering of catalytically active nanofibrous materials by electrospinning, of which most require either organic solvents, additional reducing agents or heat treatment, and hence, the green and one-step synthesis of catalytically active nanofibers with embedded noble metal nanoparticles is highly desirable.

Among noble nanoparticles, palladium nanoparticles (Pd-NPs) have gained great interest due to their high catalytic activity and inherent stability against chemical corrosion so that they can

\footnotetext{
${ }^{a}$ Institute of Materials Science \& Nanotechnology, Bilkent University, 06800 Ankara, Turkey

${ }^{b}$ Department of Fiber Science and Apparel Design, College of Human Ecology, Cornell University, Ithaca, NY, 14853, USA. E-mail: tu46@cornell.edu $\dagger$ Electronic supplementary information (ESI) available: XRD patterns of the nanofibers and a table related to the catalytic performance of the nanofibers. See DOI: 10.1039/c8nj05133j
}

maintain their physical properties for a long period of time..$^{7-9}$ They have been incorporated into various nanofiber systems through different approaches, and the resultant nanofibers have been implemented for a wide range of applications. In this regard, Demir et al. reported Pd-NP loaded poly(acrylonitrile-co-acrylic acid) nanofibers using hydrazine as a reducing agent for the nanoparticle synthesis. ${ }^{10}$ Likewise, Tong et al. reported Pd/Ag-NP embedded polyacrylonitrile (PAN) nanofibers using ammonium borate as a reducing agent. ${ }^{11}$ Pd-NP functional nanofibers were also synthesized by the thermal treatment of electrospun nanofibers, which were produced by the electrospinning of a mixed solution of PAN and palladium(II) acetate $\left(\mathrm{Pd}(\mathrm{OAc})_{2}\right) .{ }^{12}$ The electrospinning and following carbonization led to the synthesis of Pd-NP embedded carbon nanofibers, which were used for the reduction of $\mathrm{H}_{2} \mathrm{O}_{2}$ and oxidation of NADH. Tang and co-workers reported the synthesis of Pd-NPs using polydopamine-coated PAN fibers as the reducing agent. ${ }^{13}$ The Pd-NP decorated fibers were used for trichloroethene dichlorination, and excellent dichlorination performance was observed with high recyclability. Wen et al. reported a silica-supported Pd nanofiber catalyst system. ${ }^{4}$ The electrospinning of a mixed solution of poly(vinyl pyrrolidone) (PVP), tetraethyl orthosilicate, and $\mathrm{PdCl}_{2}$ and following calcination led to $\mathrm{Pd} / \mathrm{PVP} / \mathrm{SiO}_{2}$ composite nanofibers, which were used for the efficient hydrogenation of acrylic acid. As seen in the above 
examples, most of the concepts use either an additional reducing agent or heat treatment as a driving force for the synthesis of Pd-NPs.

Here we describe a facile and green synthesis route relying on the aqueous solutions of CD for the fabrication of Pd-NP embedded polymer-free CD nanofibers. The Pd-NP embedded polymer-free CD nanofibers were also produced from DMF solutions. To the best of our knowledge, this study, for the first time, reports the facile and green synthesis of Pd-NPs through the reduction of $\mathrm{CD}$ molecules and their incorporation into electrospun polymer-free CD nanofibers in which CD acts as a stabilizer for the Pd-NPs against aggregation and also CD by itself functions as a self-standing nanofibrous support matrix for Pd-NPs.

CDs are cyclic oligosaccharides with a toroidal shape bearing primary and secondary alcohol groups. Furthermore, CDs are natural, edible and biocompatible compounds and have been used for a wide spectrum of applications owing to their complexation ability with numerous hydrophobic molecules. ${ }^{14-18}$ CDs with pendant hydroxyl moieties catalyze the reduction of $\mathrm{Pd}^{2+}$ to metallic $\mathrm{Pd}^{0}$, resulting in Pd-NPs. Furthermore, CDs could be electrospun into nanofibers without the requirement of any polymeric carrier ${ }^{19}$ so that the resultant fibrous materials provide a high active CD content, and with that, they can serve as carriers of functional molecules for possible applications in drug delivery and be involved in the scavenging of environmental micropollutants. ${ }^{19,20}$ Thus, a system containing CDs would be a rational approach for the engineering of Pd-NPs embedded in functional electrospun nanofibers without the requirement of any other additives.

In this paper, the precursor $\mathrm{Pd}(\mathrm{OAc})_{2}$ was mixed with an aqueous or DMF solution of hydroxypropyl-beta-cyclodextrin (HP- $\beta$-CD) molecules and electrospun into bead-free HP- $\beta$-CD nanofibers with embedded in situ formed Pd-NPs. The nanofibers were characterized based on their morphology by SEM, and the structure and crystallinity of the nanoparticles were explored by TEM, STEM and SAED analyses. XPS was used to confirm the presence of Pd-NPs near to the nanofiber surface and explore the atomic states of Pd. Lastly, the catalytic performance of the nanocomposite nanowebs was explored by the reduction of $p$-nitrophenol (PNP) to $p$-aminophenol (PAP).

\section{Experimental section}

\subsection{Chemicals and materials}

Hydroxypropyl- $\beta$-cyclodextrin (HP- $\beta$-CD) with a substitution degree between 0.6 and 0.9 was obtained as a gift from Wacker Chemie AG (Germany). Palladium(II) acetate trimer $\left(\mathrm{C}_{12} \mathrm{H}_{18} \mathrm{O}_{12} \mathrm{Pd}_{3}\right.$, Pd 45.9-48.4\%, Alfa Aesar), $N, N$-dimethylformamide (DMF, Riedel) and sodium hydroxide $(\mathrm{NaOH}$, Fluka, $\geq 98 \%)$ were purchased. p-Nitrophenol (PNP, 99\%) was purchased from Alfa Aesar. Deionized water was produced using a Milli-Q ultrapure water system (resistivity $\geq 18 \mathrm{M} \Omega \mathrm{cm}$ ).

\subsection{Electrospinning of nanocomposite cyclodextrin nanofibers}

Nanocomposite HP- $\beta$-CD nanofibers were prepared by the electrospinning of HP- $\beta-C D$ in aqueous and DMF solutions at different concentrations, i.e., $120 \%(\mathrm{w} / \mathrm{v})$ for DMF and $160 \%(\mathrm{w} / \mathrm{v})$ for aqueous systems, which are the optimum concentrations for the production of bead-free HP- $\beta$-CD nanofibers from the respective solvent system. ${ }^{19}$ Elemental Pd contents were adjusted as 1 and 2 wt\% with respect to $\mathrm{CD}$ content. The solution $\mathrm{pH}$ was adjusted between 8.5 and 9 with $1 \mathrm{M} \mathrm{NaOH}$ and mixed overnight to obtain homogeneous dispersions of the Pd-NPs in the respective solvent system. As the reaction proceeds, the color of the solution turned black, demonstrating the formation of Pd-NPs. Thereafter, the solutions were loaded into plastic syringes having disposable metallic needles with an inner diameter of $0.6 \mathrm{~mm}$. The syringes were placed on a syringe pump (Model: SP 101IZ, WPI). The electrode of a high voltage power supply (Matsusada precision, AU Series) was clamped to the metallic needle tip. Electrospinning was performed using the following parameters: the applied voltage was between 10 and $15 \mathrm{kV}$, the tip-to-collector distance was in the range of $10-20 \mathrm{~cm}$, and the flow rate was set to $0.5 \mathrm{~mL} \mathrm{~h}^{-1}$. The electrospun nanofibers were deposited on a grounded stationary metal collector covered by a piece of aluminum foil. The electrospinning apparatus was enclosed in a Plexiglas box, and electrospinning was carried out at $25{ }^{\circ} \mathrm{C}$.

\subsection{Characterization methods}

Viscosity measurements were performed using an Anton Paar Physica MCR 301 rheometer equipped with a Peltier device for temperature control. The top plate (cone plate $\left(\mathrm{CP}, 1^{\circ}\right)$, diameter $(D) 40 \mathrm{~mm}$ ) was set at a distance of $164 \mu \mathrm{m}$ prior to the measurements. A solvent trap system was used to minimize water evaporation during rheological testing. Furthermore, the sample perimeter was covered with a low-viscous silicone oil to prevent evaporation of water during the measurements. Conductivity measurements were carried out using a conductivity meter (Mettler Toledo) at room temperature. The morphology of the electrospun nanofibers was explored by scanning electron microscopy (Quanta 200 FEG, FEI). The samples were sputtered with a thin layer of $\mathrm{Au}(\sim 5 \mathrm{~nm})$ using a Gatan 682 Precision Etching and Coating System (PECS), and the mean diameters $(\langle D\rangle)$ of the spun fibers and their size distributions were calculated by analyzing $c a .100$ fibers from the SEM images using Image (NIH, Bethesda, MD, USA). Transmission electron microscopy was performed at $300 \mathrm{kV}$ using a FEI Tecnai G2F30 microscope. In this regard, the nanocomposite solutions were directly electrospun on TEM grids. Wide-angle X-ray diffraction tests were carried out using a PANalytical X'Pert Pro MPD, which was powered by a Philips PW3040/60 X-ray generator and fitted with an X'Celerator detector. X-Rays were generated from a $\mathrm{Cu}$ anode supplied with a voltage of $40 \mathrm{kV}$ and a current of $40 \mathrm{~mA}$. The data were collected in the $2 \theta$ range of $5-90^{\circ}$ using a scanning X'Celerator detector and analyzed using PANalytical High Score Plus software (version 2.0). An X-ray photoelectron spectrometer (XPS) (Thermo Scientific) was used to study the chemical state of Pd in the nanocomposite nanofibers. XPS was performed by means of a flood gun charge neutralizer system equipped with an $\mathrm{Al} \mathrm{K}-\alpha$ X-ray source $(h \nu=1486.6 \mathrm{eV})$. High-resolution spectra were recorded for the spectral regions of Pd with a pass energy of $50 \mathrm{eV}$. FTIR spectra 
were recorded on a Bruker-VERTEX 70 spectrophotometer. The samples were blended with $\mathrm{KBr}$ and pressed into transparent pellets. A total of 64 scans were taken per sample with a resolution of $4 \mathrm{~cm}^{-1}$.

\subsection{Catalytic activity tests}

The catalytic performance of the nanocomposite HP- $\beta$-CD nanofibers was explored by the reduction of a nitroarene compound, i.e., $p$-nitrophenol (PNP) to $p$-aminophenol (PAP), in the presence of $\mathrm{NaBH}_{4}$, and the reaction was monitored by UV-vis measurements. In a typical reaction test, the freshly prepared sodium borohydride $\left(\mathrm{NaBH}_{4}, 1.0 \mathrm{~mL}\right.$ of $\left.7 \mathrm{mM}\right)$ solution was mixed with $1.7 \mathrm{~mL}$ of $0.1 \mathrm{mM}$ PNP in a standard quartz cell with a path length of $1 \mathrm{~cm}$. After the addition of the nanofibers ( $1 \mathrm{mg}$ ), the first UV-vis absorption spectrum was recorded in $15 \mathrm{~s}$, and the following spectra were collected every $60 \mathrm{~s}$ in the wavelength range of $200-550 \mathrm{~nm}$. As control experiments, the conversion of PNP was explored in the presence of Pd-free HP- $\beta$ CD fibers and $\mathrm{NaBH}_{4}$ or in the presence of only $\mathrm{NaBH}_{4}$. The reusability experiment of the Pd-NPs in the HP- $\beta-\mathrm{CD} / \mathrm{Pd}-\mathrm{NP}$ nanofibers was performed by the addition of $0.17 \mathrm{~mL}$ from $1 \mathrm{mM}$ PNP solution. The conversion was followed by UV-vis analysis until the peak at $400 \mathrm{~nm}$ disappeared, and afterwards, $0.17 \mathrm{~mL}$ of PNP was added to the cuvette, and this cycle was repeated three times.

\section{Results and discussion}

The nanocomposite HP- $\beta$-CD nanofibers were prepared by the electrospinning of HP- $\beta$-CD from aqueous and DMF solutions containing two different $\mathrm{Pd}$ contents ( 1 and $2 \mathrm{wt} \%$ with respect to $\mathrm{CD}$ ). In this regard, HP- $\beta$-CD molecules were treated with $\mathrm{Pd}(\mathrm{OAc})_{2}$ overnight while the color of the solution turned black, indicating the formation of Pd-NPs. The characteristics of the HP- $\beta$-CD solutions containing 1 and $2 \mathrm{wt} \% \mathrm{Pd}$ are given in Table 1 . The viscosity of the HP- $\beta$-CD solutions $(120 \%(\mathrm{w} / \mathrm{v}))$ in DMF was determined to be 0.55 and $0.68 \mathrm{~Pa}$ s for 1 and $2 \mathrm{wt} \%$ Pd loadings, respectively. On the other hand, a lower viscosity was observed for aqueous HP- $\beta$-CD solutions $(160 \%(\mathrm{w} / \mathrm{v}))$ : the respective values were 0.38 and $0.30 \mathrm{~Pa} \cdot \mathrm{s}$ for 1 and $2 \mathrm{wt} \% \mathrm{Pd}$ loadings. Although the concentration of HP- $\beta$-CD in DMF was lower than that in aqueous solutions, the higher viscosity of HP- $\beta$-CDs in DMF can be attributed to enhanced intermolecular associations. A similar trend was reported in Pd-free CD solutions where a higher viscosity was observed for the solutions of HP- $\beta$-CD molecules in DMF than in water. ${ }^{19}$ The conductivity of the respective solutions increased with higher Pd concentration. The conductivity of the HP- $\beta$-CD/Pd-NPs- $1 \%$ was determined to be $34 \mu \mathrm{S} \mathrm{cm}^{-1}$, while it increased two-fold to $61.7 \mu \mathrm{S} \mathrm{cm}^{-1}$ with an increasing Pd content. On the other hand, the conductivity values were much higher for water-based systems. The respective values were determined to be 517 and $1111 \mu \mathrm{S} \mathrm{cm}^{-1}$ for 1 and 2 wt\% Pd loadings.

The nanocomposite nanofibers of HP- $\beta-C D$ and Pd-NPs were produced by electrospinning. The SEM images of the nanofibers revealed bead-free nanofibers whose size, regardless of the solvent system used, decreased with a higher Pd loading: for the nanofibers produced from DMF solutions, the mean nanofiber diameter slightly decreased from 195 to $190 \mathrm{~nm}$ with an increased Pd loading from 1 to $2 \mathrm{wt} \%$ (Fig. 1). Likewise, for the nanofibers electrospun from aqueous solutions, the mean diameters of the nanofibers were determined to be 530 and $420 \mathrm{~nm}$ for 1 and $2 \mathrm{wt} \%$ Pd loadings, respectively. When lower HP- $\beta$-CD concentrations were used for the electrospinning, the resultant nanofibers were obtained with smaller diameters than those electrospun from aqueous solutions (i.e., $c_{\mathrm{HP}-\beta-\mathrm{CD}}=$ $160 \mathrm{wt} \%$ for water and $c_{\mathrm{HP}-\beta-\mathrm{CD}}=120 \mathrm{wt} \%$ in DMF). On the other hand, Celebioglu et al. reported a reverse trend for pure HP- $\beta$ CD nanofibers produced from aqueous and DMF solutions: for the nanofibers electrospun from DMF solutions at $120 \%(\mathrm{w} / \mathrm{v})$ HP- $\beta$-CD, the mean fiber diameter was found to be $1125 \pm$ $360 \mathrm{~nm}$ while the respective value for water-based systems at $160 \%(\mathrm{w} / \mathrm{v}) \mathrm{HP}-\beta-\mathrm{CD}$ was determined to be $745 \pm 370 \mathrm{~nm} .{ }^{19} \mathrm{~A}$ decrease in the nanofiber diameter with an increased $\mathrm{Pd}$ loading can be ascribed to the enhanced conductivity of the electrospinning solutions. The structure and distribution of the Pd-NPs were analyzed by TEM imaging. Fig. 2 shows the TEM images of the nanofibers where uniform Pd-NPs were homogenously distributed in the nanofiber matrix with a mean particle size varying between 3.7 and $4.9 \mathrm{~nm}$ depending on the solvent system and Pd loading. For the nanofibers produced from DMF solutions at $1 \mathrm{wt} \%$ Pd loading, the mean particle size was calculated to be $3.7 \pm 1.0 \mathrm{~nm}$ and $4.0 \pm 1.1 \mathrm{~nm}$ for $2 \mathrm{wt} \% \mathrm{Pd}$ loading. On the other hand, the Pd-NPs produced from aqueous solutions were slightly bigger in size: the mean particle size was found to be $4.7 \pm 1.1 \mathrm{~nm}$ for HP- $\beta$-CD/Pd-NPs- $1 \%$ and $4.9 \pm$ $1.2 \mathrm{~nm}$ for HP- $\beta$-CD/Pd-NPs-2\%. For both solvent systems, increasing Pd loading led to the formation of larger Pd-NPs. The examination of the crystal structure of the Pd-NPs by highresolution TEM (HRTEM) revealed lattice fringes (Fig. 3). For the Pd-NPs produced in DMF, the respective HRTEM image showed that the interplanar spacing of the Pd-NP lattice was found to be $0.224 \mathrm{~nm}$, which coincides well with the $\{111\}$ lattice spacing of a face-centered cubic (fcc) Pd. ${ }^{21}$ Furthermore, the crystalline structure of the Pd-NPs in nanofibers was analyzed by wideangle XRD analysis, which revealed small peaks at 40.5 and

Table 1 The characteristics of HP- $\beta-\mathrm{CD} / \mathrm{Pd}$ solutions and their nanofibers produced at different Pd loadings

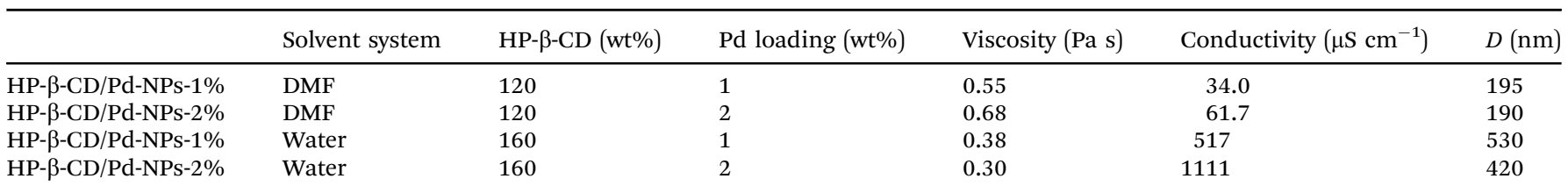



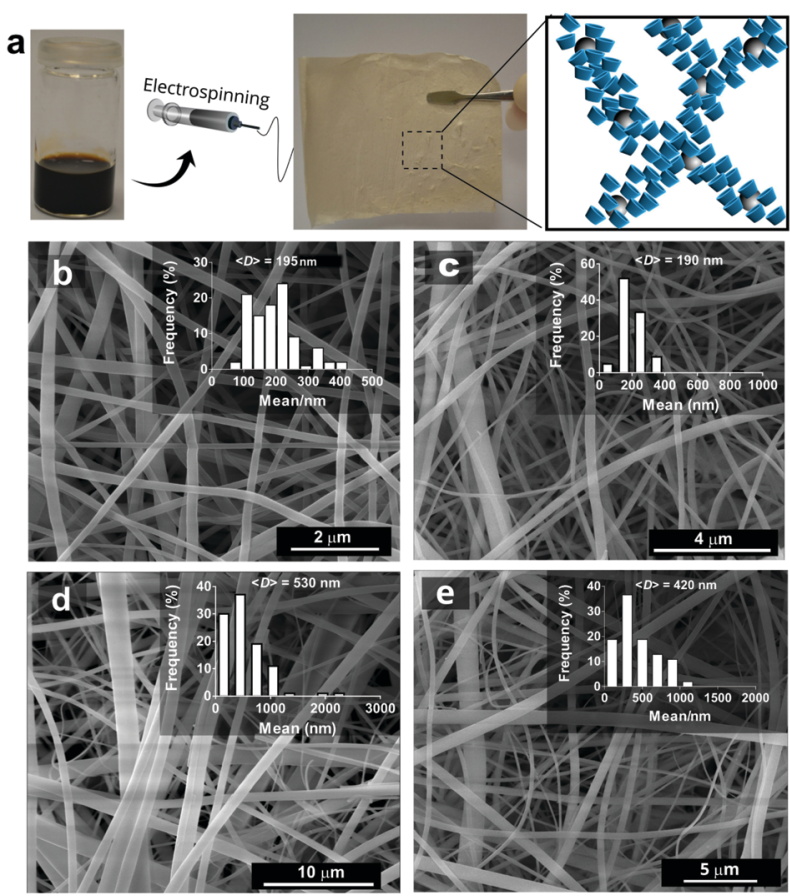

Fig. 1 (a) Optical photos of the HP- $\beta$-CD/Pd-NP dispersion after overnight mixing and the nanofiber mat produced by electrospinning. A cartoon illustration of the HP- $\beta-C D / P d-N P$ nanofibers with in situ formed Pd-NPs. The scanning electron micrographs of the Pd-NPs embedded in HP- $\beta$-CD nanofibers produced from DMF solutions at $1 \mathrm{wt} \%$ (b) and $2 \mathrm{wt} \%$ (c) Pd loadings. The SEM images of the Pd-NP functional HP- $\beta-C D$ nanofibers generated from aqueous solutions at 1 wt\% (d) and 2 wt\% (e) Pd loadings. Insets show the size distribution plots of the respective nanofibers.

$47.3^{\circ}$ due to the $\{111\}$ and $\{200\}$ planes of metallic Pd (Fig. S1, ESI $\dagger$ ). Thus, the appearance of the dominantly exposed (111) facets suggests the presence of a highly active surface on the particles. The selected-area electron diffraction (SAED) analysis of the samples demonstrated the presence of diffuse rings arose from the $\{111\},\{200\},\{220\}$ and $\{311\}$ reflections. The appearance of various planes demonstrates their polycrystalline structure, and the dominant exposed plane of $\{111\}$ suggests the presence of anisotropy in shape. ${ }^{22}$ XPS experiments were conducted to confirm the presence of the Pd-NPs near the fiber surface, and the reduction level of $\mathrm{Pd}$ in its chemical state was evaluated. Fig. 4 shows the deconvoluted Pd 3d XPS spectra of the nanofibers produced using different solvent systems and Pd loadings. The XPS spectrum of Pd $3 d$ showed a doublet of a low-energy Pd $3 \mathrm{~d}_{5 / 2}$ band and a high-energy Pd $3 \mathrm{~d}_{3 / 2}$ band. The corresponding binding energy values (i.e., $335.7 \mathrm{eV}$ for $\mathrm{Pd} 3 \mathrm{~d}_{5 / 2}$ and $341.6 \mathrm{eV}$ for $\mathrm{Pd} 3 \mathrm{~d}_{3 / 2}$ ) for Pd $3 \mathrm{~d}$ agree well with those of previous studies. ${ }^{23-25}$ Furthermore, these bands are composed of two pairs of doublets belonging to metallic $\mathrm{Pd}^{0}$ and oxidized $\mathrm{Pd}^{2+}$. The proportion of $\mathrm{Pd}^{0}$ was dominant for all samples, suggesting the higher proportion of metallic Pd on the nanofibers owing to its efficient reduction by $\mathrm{CD}$ molecules.

The proportions of $\mathrm{Pd}^{0} / \mathrm{Pd}^{2+}$ were found to be 1.87 and 1.80 , for the Pd-NPs synthesized from DMF solutions with 1 and $2 \mathrm{wt} \%$ Pd loading, and the respective values were calculated to be 1.70 and 1.45 for the Pd-NPs produced from aqueous solutions.
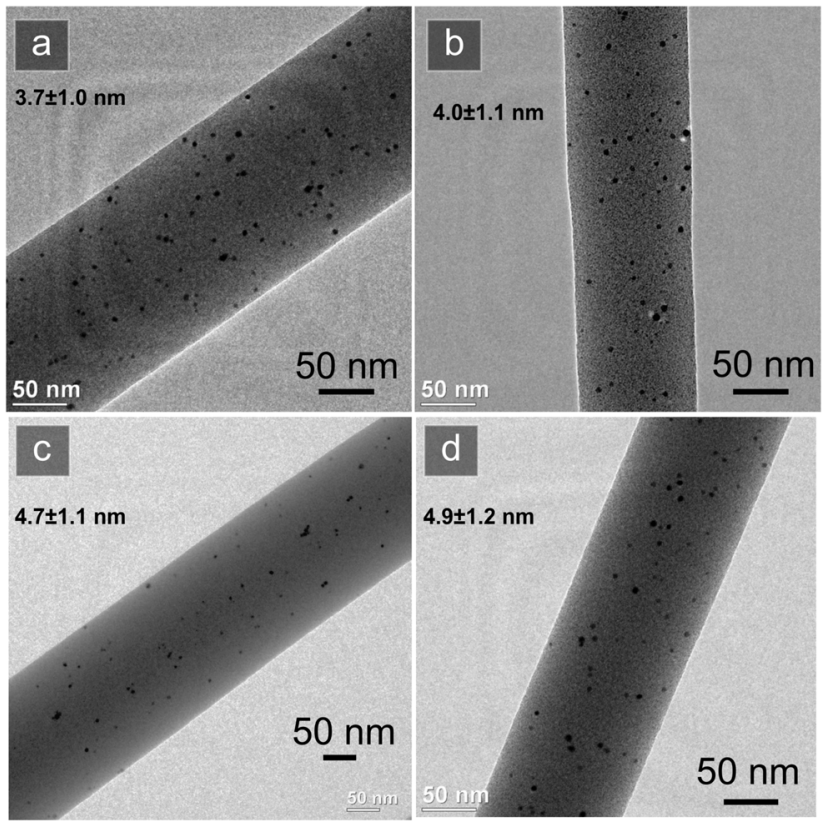

Fig. 2 The TEM images of the HP- $\beta$-CD/Pd-NP nanofibers prepared at different formulations. The nanofibers electrospun from DMF ( $a$ and $b$ ) and aqueous solutions ( $c$ and $d$ ) at the $1 \mathrm{wt} \%$ ( $a$ and $c$ ) and $2 \mathrm{wt} \%$ (b and d) Pd-NPs concentrations, respectively.

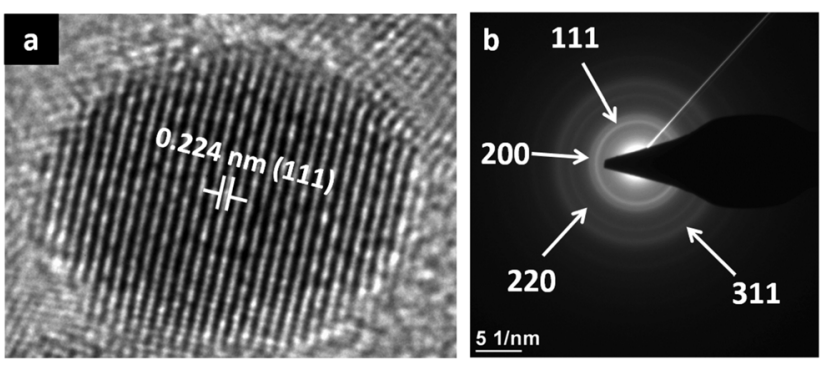

Fig. 3 (a) The HRTEM image of the Pd-NPs loaded HP- $\beta$-CD nanofibers generated from DMF solutions, and (b) the corresponding SAED pattern obtained from the nanofibers.

The interaction between the Pd-NPs and HP- $\beta$-CD was explored by FTIR analysis. Fig. 5 shows the FTIR spectra of the nanocomposite nanofibers produced from DMF and aqueous solutions with two different Pd loadings ( 1 and 2 wt\%). For the nanocomposite nanofibers, a peak appeared at $1730 \mathrm{~cm}^{-1}$ owing to the stretching vibration of the carbonyl $(\mathrm{C}=\mathrm{O})$ group in the $\mathrm{Pd}(\mathrm{OAc})_{2}$ (Fig. 5a and c). This was observed for both nanocomposite nanofibers produced from aqueous and DMF solutions, and their intensity increased with a higher Pd loading. The formed Pd-NPs interacted with the hydroxyl groups of CD and caused an apparent frequency shift of $\mathrm{OH}$ stretching of the $\mathrm{CD}$ molecules (Fig. 5b and d). The peak shifted to higher frequencies for the nanofibers generated from DMF and aqueous solutions due to the presence of physical interactions between CD and Pd-NPs. This agrees with a previous report, which showed the shift of $\mathrm{OH}$ stretching of the CD molecules due to the interactions between $\mathrm{CD}$ and nanoparticles (Ag-NPs and Au-NPs). ${ }^{26,27}$ Pd-NPs are 

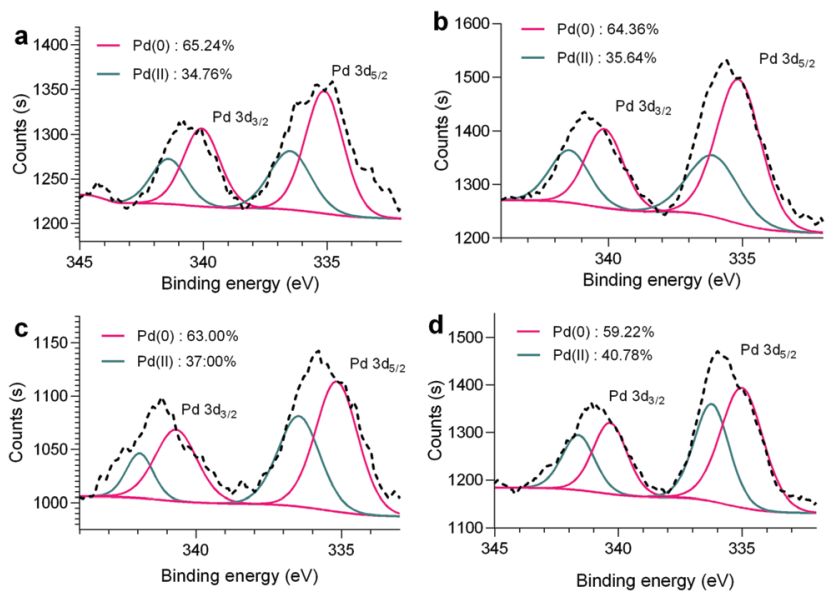

Fig. 4 The deconvoluted Pd $3 d$ XPS spectra of the HP- $\beta-C D$ nanofibers generated at different formulations. HP- $\beta-C D / P d-N P$ nanofibers produced from DMF solutions containing 1 wt\% (a) and 2 wt\% (b) Pd. HP- $\beta-C D / P d-N P$ nanofibers produced from aqueous solutions containing $1 \mathrm{wt} \%$ (c) and 2 wt\% (d) Pd.


Fig. 5 FTIR spectra of the HP- $\beta-C D / P d-N P$ nanofibers produced from different solvent systems (water and DMF) at two different Pd loadings ( 1 and 2 wt\%), along with pure HP- $\beta$-CD fibers. The high resolution XPS of the electrospun HP- $\beta-C D / P d-N P$ nanofibers produced from two solvent systems (DMF and water) at different Pd-NPs loadings (1 and 2 wt\%).

known to have high catalytic activity for a wide spectrum of reactions, including carbon-carbon cross-coupling, hydrogenation and dehydrogenation. ${ }^{28-31}$ The incorporation of the Pd-NPs into the nanofibers transforms them into catalytically active nanomaterials. In this regard, numerous studies on Pd-NP functional nanofiber systems have been reported for different catalytic reactions, including the photocatalysis of methylene blue, ${ }^{32}$ Heck coupling reactions between iodobenzene and acrylates, ${ }^{33}$ and hydrogenation of aldehydes ${ }^{34}$ and $p$-nitrophenol. ${ }^{35} p$-Nitrophenol is a nitroarene-type of molecule and can be found in wastewater, ${ }^{36}$ and can damage the central nervous system, liver and kidneys. ${ }^{37}$ It is thus considered as a pollutant by the U.S. Environmental Protection Agency (EPA). ${ }^{38}$
Due to its high stability and solubility in water, it can be converted into a less harmful and biodegradable derivative by converting the nitro groups to amine. In this regard, several studies have reported the reduction of $p$-nitrophenol to $p$-aminophenol using silver nanoparticles immobilized on fibrous nanosilica particles, ${ }^{39} \mathrm{Ni@Pd} \mathrm{core-shell} \mathrm{nanoparticle} \mathrm{modified} \mathrm{fibrous}$ silica nanospheres ${ }^{40}$ metal organic framework-derived magnetic porous carbon composite-supported gold and palladium nanoparticles. ${ }^{41}$

The catalytic activity of the Pd-NP-embedded CD nanofibers was explored by the reduction of $p$-nitrophenol (PNP) to $p$-aminophenol (PAP). In this context, the nanofibers were treated with a PAP solution over a period of time, and the absorbance was followed by UV-vis measurements with 1 min intervals (Fig. 6). As a co-catalyst, $\mathrm{NaBH}_{4}$ was used as an additive during the
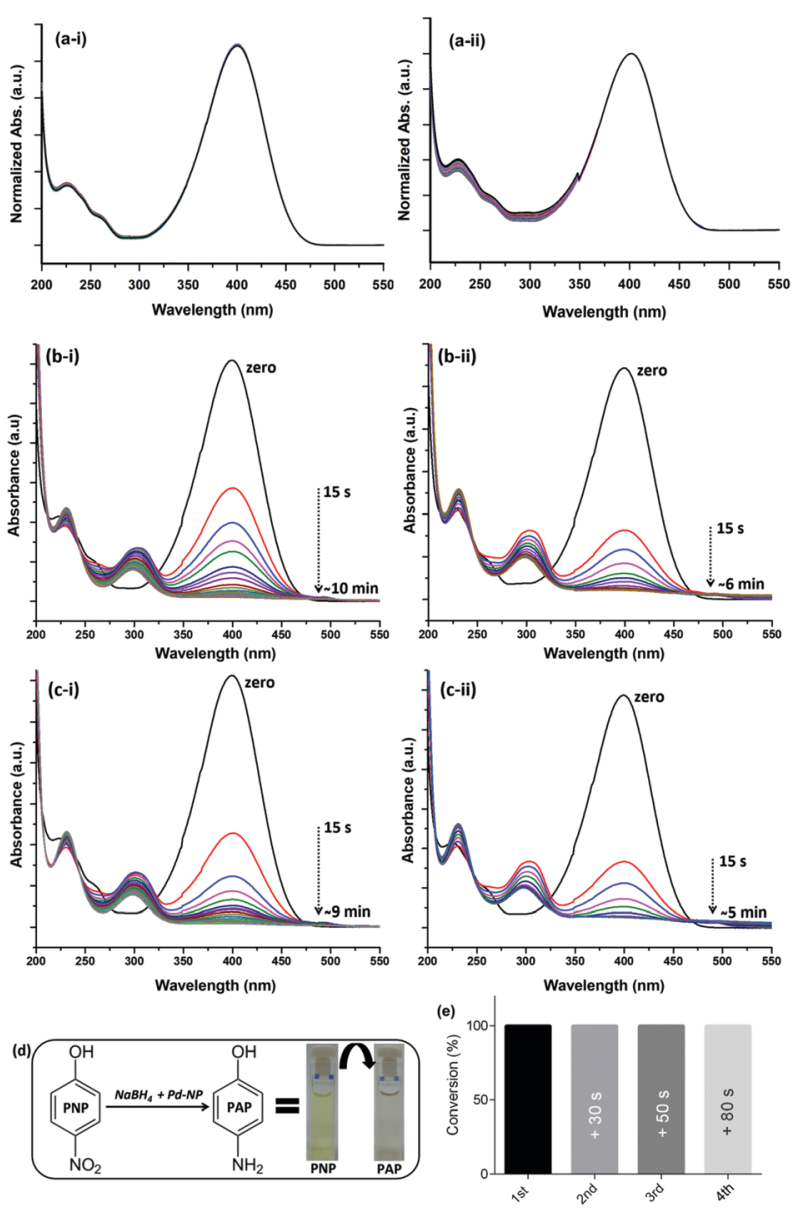

Fig. 6 The catalytic performance of the HP- $\beta-C D / P d-N P$ nanofibers over the reduction of PNP to PAP. (a-i) UV-vis spectra of PNP in the presence of only $\mathrm{NaBH}_{4}$ and the absence of fiber. (a-ii) UV-vis spectra of PNP in the presence of $\mathrm{NaBH}_{4}$ and Pd-free HP- $\beta-C D$ fibers. (b-i) UV-vis spectra of PNP solution treated with HP- $\beta-C D / P d-N P s-1 \%-D M F$, (b-ii) HP- $\beta-C D /$ Pd-NPs-2\%-DMF, (c-i) HP- $\beta$-CD/Pd-NPs-1\%-water and (c-ii) HP- $\beta$-CD/ Pd-NPs-2\%-water nanofibers. (d) The reaction mechanism of the reduction of PNP to PAP by $\mathrm{NABH}_{4}$ and Pd-NPs, and the respective optical photos of the PNP solution before and after fiber treatment. (e) The reusability of the Pd-NPs over the conversion of PNP. The extra time needed for the complete conversion of PNP was written on the respective column. 
experiments. There was no clear decrease in the absorption peak of PNP in the presence of Pd-free HP- $\beta$-CD fibers or only $\mathrm{NaBH}_{4}$ (Fig. 6a and b), suggesting that no hydrogenation reaction took place in the absence of Pd-NPs in a given period of time. Normally, PNP shows a maximum absorbance at $317 \mathrm{~nm}$, which shifted to $400 \mathrm{~nm}$ with the addition of $\mathrm{NaBH}_{4}$ due to the formation of phenolate ions. After the addition of the nanofibers, a decrease in the intensity of the absorption peak at $400 \mathrm{~nm}$ was observed while a new peak appeared at $300 \mathrm{~nm}$ owing to the formation of $p$-aminophenol (PAP). The decrease was faster for the nanofibers with $2 \mathrm{wt} \%$ Pd loadings than those with $1 \mathrm{wt} \% \mathrm{Pd}$ loading. This was the case for nanofibers produced from aqueous solutions or DMF: a higher Pd content increased the reaction kinetics and led to the formation of PAP in less time. For the nanofibers generated from DMF solutions, the complete conversion of PNP to PAP took about $10 \mathrm{~min}$ for the $1 \mathrm{wt} \%$ Pd loading while this duration decreased two-fold to 6 min with increasing Pd content. A similar trend was observed for the composite nanofibers produced from aqueous solutions. The nanofibers with $1 \mathrm{wt} \%$ Pd content converted PNP to PAP in 9 min while it decreased to 5 min with an increasing Pd content to $2 \mathrm{wt} \%$. The reaction mechanism is shown in Fig. $6 \mathrm{~d}$, in which the optical photos of the PNP solution before and after treatment with the nanocomposite nanofibers are shown. The yellowish solution turned colorless in a few minutes with the treatment of the nanocomposite nanofibers, demonstrating the high catalytic activity of the HP- $\beta$-CD/Pd-NP nanofiber system. Assuming that all added Pd content remained in the resultant nanofibers, the TOF values were close to each other and the TOF values were calculated to be 10.17 and $12.25 \mathrm{~h}^{-1}$ for the HP- $\beta-\mathrm{CD} / \mathrm{Pd}-\mathrm{NP}$ nanofibers prepared from DMF and aqueous solutions, respectively. The respective values were compared with the Pd-NPs on different supports (Table S1, ESI $\dagger$ ). Although there are some systems with better catalytic activity, they are mainly based on conductive supports or with different metals. Nonetheless, the catalytic activity of the HP- $\beta$-CD/Pd-NPs systems for the reduction of PNP is comparable with those of the $\mathrm{ZnO}$ supported Pd nanoparticles $(\mathrm{Pd} / \mathrm{ZnO})^{42}$ and Pd-decorated mesoporous $\mathrm{SiO}_{2}$ SBA-15 nanoparticles. ${ }^{43}$

Here, we have shown that HP- $\beta$-CD molecules not only acted as both a reducing agent and a stabilizer, they also served as a handy nanofibrous carrier matrix in the form of a self-standing mat for the Pd-NPs while keeping the nanoparticles active and stable over a period of time and preventing their aggregation. The HP- $\beta$-CD/Pd-NP nanofibers are electrospun from their aqueous solution and DMF, so, the HP- $\beta$-CD/Pd-NP nanofibrous mats have a fast-dissolving characteristic which is due to the highly water-soluble nature of CDs. The HP- $\beta-\mathrm{CD} / \mathrm{Pd}-\mathrm{NP}$ nanofibrous mats are self-standing and very handy, being very light-weight, and they do not occupy high volume and are quite transportable when compared to nanoparticles which are kept in their solutions. Here, when HP- $\beta-\mathrm{CD} / \mathrm{Pd}-\mathrm{NP}$ nanofibrous mats are placed in a PNP aqueous solution, the nanofibrous matrix readily dissolves and the Pd-NPs present in the HP- $\beta-\mathrm{CD} /$ Pd-NP nanofiber matrix become active for catalytic reactions. Furthermore, the reusability of the Pd-NPs has also been shown here once the HP- $\beta$-CD/Pd-NP nanofiber matrix was placed and dissolved in an aqueous PNP solution for the first catalytic reaction, and the system can be reused without any drastic difference in the catalytic performance. The reusability experiments were performed with the addition of an identical PNP content from the concentrated PNP solution (1 mM). Fig. 6 shows the results related to the reusability of the Pd-NPs over the conversion of the PNP added. After the addition of fresh PNP, UV-vis spectra were obtained at the onset of the reaction. The results revealed that the complete conversion of PNP took place, but the time needed for the total conversion of PNP increased after each use as shown in Fig. 6e. Overall, the present concept allows the one-step synthesis of Pd-NPs through reduction by CDs without the need of any other reducing agent. Furthermore, the same mixture can directly be electrospun into bead-free nanofibers by electrospinning. In addition to the reducing role of the CD molecules, they also stabilize the Pd-NPs, and hence, the respective electrospun nanowebs offer many advantages, including the long-term storage of Pd-NPs, being lightweight and transportable and having a handy structure.

\section{Conclusion}

For the first time, polymer-free nanocomposite electrospun nanofibers of HP- $\beta$-CD and the in situ formed Pd-NPs have been reported. HP- $\beta$-CD acted as the reducing agent and gave rise to the formation of Pd-NPs by the reduction of $\mathrm{Pd}^{2+}$ to metallic $\mathrm{Pd}^{0}$ without the requirement of any other reducing agent. The nanocomposite solutions were successfully transformed into bead-free $\mathrm{HP}-\beta-\mathrm{CD} / \mathrm{Pd}-\mathrm{NP}$ nanofibers by electrospinning. The green synthesis of the Pd-NPs in water and their electrospinning led to larger nanofibers with mean diameters of 530 and $420 \mathrm{~nm}$ for 1 and $2 \mathrm{wt} \%$ Pd loadings while the respective values for the DMF-based system were found to be 195 and $190 \mathrm{~nm}$. A similar trend was observed in the size of the formed Pd-NPs: the NPs synthesized in water were slightly larger in size. The TEM and HRTEM images of the Pd-NPs revealed the homogenously distributed Pd-NPs in the size range of 3.7-4.9 nm depending on the solvent-type and Pd loading. XPS confirmed the presence of a higher proportion of metallic $\mathrm{Pd}^{0}$ than $\mathrm{Pd}^{2+}$, demonstrating the successful reduction of the Pd atoms to form nanoparticles with the help of HP- $\beta$-CD molecules. The catalytic activity of the nanofibers was explored by the reduction of $p$-nitrophenol (PNP), and the results showed high catalytic performance of the nanocomposite nanofibers, which could reduce PNP in few minutes into $p$-aminophenol (PAP). Overall, this paper describes a one-step facile and green route for the synthesis of ultrasmall Pd-NPs with sizes in the 3-5 $\mathrm{nm}$ range through reduction by renewable $\mathrm{CD}$ molecules, and their transformation into nanofibers through electrospinning for catalytic applications.

\section{Conflicts of interest}

There are no conflicts to declare. 


\section{References}

1 Electrospun materials for tissue engineering and biomedical applications: research, design and commercialization, ed. T. Uyar and E. Kny, Elsevier, Woodhead Publishing, 2017.

2 J. Wang and M. Windbergs, Eur. J. Pharm. Biopharm., 2017, 119, 283-299.

3 M. T. Hunley and T. E. Long, Polym. Int., 2007, 57, 385-389.

4 S. Wen, M. Liang, R. Zou, Z. Wang, D. Yue and L. Liu, RSC Adv., 2015, 5, 41513-41519.

5 B. Coşkuner Filiz and A. Kantürk Figen, Int. J. Hydrogen Energy, 2016, 41, 15433-15442.

6 J. Xue, J. Xie, W. Liu and Y. Xia, Acc. Chem. Res., 2017, 50, 1976-1987.

7 E. D. Sultanova, V. V. Salnikov, R. K. Mukhitova, Y. F. Zuev, Y. N. Osin, L. Y. Zakharova, A. Y. Ziganshina and A. I. Konovalov, Chem. Commun., 2015, 51, 13317-13320.

8 I. Saldan, Y. Semenyuk, I. Marchuk and O. Reshetnyak, J. Mater. Sci., 2015, 50, 2337-2354.

9 A. Nirmala Grace and K. Pandian, Mater. Chem. Phys., 2007, 104, 191-198.

10 M. M. Demir, M. A. Gulgun, Y. Z. Menceloglu, B. Erman, S. S. Abramchuk, E. E. Makhaeva, A. R. Khokhlov, V. G. Matveeva and M. G. Sulman, Macromolecules, 2004, 37, 1787-1792.

11 Y. Tong, X. Lu, W. Sun, G. Nie, L. Yang and C. Wang, J. Power Sources, 2014, 261, 221-226.

12 J. Huang, D. Wang, H. Hou and T. You, Adv. Funct. Mater., 2008, 18, 441-448.

13 J. Wang, C. K. Ng, B. Cao, W. Qing, F. Liu and C. Y. Tang, Appl. Catal., A, 2018, 559, 122-126.

14 T. Nagai, in Inclusion Phenomena in Inorganic, Organic, and Organometallic Hosts, ed. J. L. Atwood and J. E. D. Davies, Springer, Netherlands, Dordrecht, 1987, pp. 29-38.

15 K. Chaturvedi, K. Ganguly, A. R. Kulkarni, V. H. Kulkarni, M. N. Nadagouda, W. E. Rudzinski and T. M. Aminabhavi, Expert Opin. Drug Delivery, 2011, 8, 1455-1468.

16 M. E. Davis and M. E. Brewster, Nat. Rev. Drug Discovery, 2004, 3, 1023.

17 R. Challa, A. Ahuja, J. Ali and R. K. Khar, AAPS PharmSciTech, 2005, 6, E329-E357.

18 E. M. M. Del Valle, Process Biochem., 2004, 39, 1033-1046.

19 A. Celebioglu and T. Uyar, Nanoscale, 2012, 4, 621-631.

20 A. Celebioglu, Z. I. Yildiz and T. Uyar, Sci. Rep., 2017, 7, 7369 .
21 X. Chen, G. Wu, J. Chen, X. Chen, Z. Xie and X. Wang, J. Am. Chem. Soc., 2011, 133, 3693-3695.

22 S. Navaladian, B. Viswanathan, T. K. Varadarajan and R. P. Viswanath, Nanoscale Res. Lett., 2008, 4, 181.

23 K. Zhang, C. Wang, D. Bin, J. Wang, B. Yan, Y. Shiraishi and Y. Du, Catal. Sci. Technol., 2016, 6, 6441-6447.

24 S. Yang, J. Dong, Z. Yao, C. Shen, X. Shi, Y. Tian, S. Lin and X. Zhang, Sci. Rep., 2014, 4, 4501.

25 Z.-R. Yang, S.-Q. Wang, J. Wang, A.-J. Zhou and C.-W. Xu, Sci. Rep., 2017, 7, 15479.

26 A. Celebioglu and T. Uyar, RSC Adv., 2013, 3, 10197-10201.

27 A. Celebioglu, F. Topuz, Z. I. Yildiz and T. Uyar, Carbohydr. Polym., 2019, 207, 417-479.

28 T. Kim, X. Fu, D. Warther and M. J. Sailor, ACS Nano, 2017, 11, 2773-2784.

29 F. P. da Silva, J. L. Fiorio and L. M. Rossi, ACS Omega, 2017, 2, 6014-6022.

30 A. Bej, K. Ghosh, A. Sarkar and D. W. Knight, RSC Adv., 2016, 6, 11446-11453.

31 L. Djakovitch, K. Köhler and J. G. D. Vries, in Nanoparticles and Catalysis, ed. D. Astruc, Wiley, 2008, ch. 10, DOI: 10.1002/9783527621323.

32 H.-G. Lee, A.-I. Gopalan, G. Sai-Anand, B.-C. Lee, S.-W. Kang and K.-P. Lee, Mater. Chem. Phys., 2015, 154, 125-136.

33 Q. Meng, J. Bai, S. Guo and C. Li, Chem. Res. Chin. Univ., 2015, 31, 1072-1077.

34 J. Yang, M. Yuan, D. Xu, H. Zhao, Y. Zhu, M. Fan, F. Zhang and Z. Dong, J. Mater. Chem. A, 2018, 6, 18242-18251.

35 K. S. Ranjith, A. Celebioglu, H. Eren, N. Biyikli and T. Uyar, Adv. Mater. Interfaces, 2017, 4, 1700640.

36 Y.-C. Chang and D.-H. Chen, J. Hazard. Mater., 2009, 165, 664-669.

37 T. Aditya, A. Pal and T. Pal, Chem. Commun., 2015, 51, 9410-9431.

38 A. Chinnappan, S. K. Eshkalak, C. Baskar, M. Khatibzadeh, E. Kowsari and S. Ramakrishna, Nanoscale Adv., 2019, 1, 305-313.

39 Z. Dong, X. Le, X. Li, W. Zhang, C. Dong and J. Ma, Appl. Catal., B, 2014, 158-159, 129-135.

40 Z. Dong, X. Le, C. Dong, W. Zhang, X. Li and J. Ma, Appl. Catal., B, 2015, 162, 372-380.

41 Z. Dong, X. Le, Y. Liu, C. Dong and J. Ma, J. Mater. Chem. A, 2014, 2, 18775-18785.

42 M.-R. Kim and S.-H. Choi, J. Nanomater., 2009, 7.

43 J. Morère, M. J. Torralvo, C. Pando, J. A. R. Renuncio and A. Cabañas, RSC Adv., 2015, 5, 38880-38891. 